\title{
Lens ageing as an indicator of tissue damage associated with smoking and non-enzymatic glycation - a twin study
}

\author{
L. Kessel ${ }^{1}$, J. L. Hougaard ${ }^{1}$, B. Sander ${ }^{1}$, K. O. Kyvik ${ }^{2}$, T. I. A. Sørensen ${ }^{3}$, M. Larsen ${ }^{1}$ \\ ${ }^{1}$ Department of Ophthalmology, Herlev Hospital, University of Copenhagen, Denmark \\ ${ }^{2}$ Danish Twin Registry, University of Southern Denmark, Odense, Denmark \\ ${ }^{3}$ Danish Epidemiology Science Center, Institute of Preventive Medicine, Copenhagen University Hospital, Denmark
}

\section{Abstract}

Aims/hypothesis. With ageing the long-lived proteins of the human lens undergo denaturation by non-enzymatic glycation. The denaturated proteins are fluorescent, a property that can be assessed in vivo by fluorometry. Our aim was to examine the relative contribution of hereditary and environmental effects on the accumulation of fluorescent compounds in the lens. Methods. We examined 59 monozygotic and 55 dizygotic healthy twin pairs recruited from a populationbased register of twins. Lens autofluorescence was measured on the undilated eye. All subjects underwent an OGTT and information on smoking habits was obtained. The genetic and environmental effects were estimated by structural equation modelling.

Results. Lens autofluorescence was related to age $\left(\mathrm{R}^{2}=53 \%\right)$, current glucose homeostasis $\left(\mathrm{R}^{2}=10 \%\right)$ and smoking habits $\left(\mathrm{R}^{2}=10 \%\right)$. After adjusting for these factors, interindividual variation in lens autofluores- cence was statistically attributable to hereditary factors by approximately $28 \%$ as well as shared environment by $58 \%$ and non-shared environment by $14 \%$. The hereditary factor seems not to be linked to a genetic predisposition to diabetes.

Conclusion/interpretation. The correlation in lens fluorescence was greater in monozygotic twins than dizygotic twins indicating a genetically predetermined susceptibility for the accumulation of fluorophores in the lens, the relative importance of which was found to increase with age. Since fluorophore formation in the lens is attributable to non-enzymatic glycation our results support that genetic characteristics to some degree determine the susceptibility to glycation related diabetes complications and ageing processes. [Diabetologia (2002) 45:1457-1462]

Keywords: Advanced glycation end products, ageing, crystalline lens, diabetes mellitus, environment, fluorometry, genetics, lens fluorescence, smoking, twins.
The crystalline lens of the eye is an infolded ectodermal structure. Its function of forming an image on the retina requires an optically clear internal structure. This is obtained by specialized cells with a high content of densely packed proteins with no apparent turnover. With time these proteins are subject to physio-

Received: 20 December 2001 / Revised: 19 June 2002

Published online: 5 September 2002

C) Springer-Verlag 2002

Corresponding author: L. Kessel, MD, Department of Ophthalmology, Herlev Hospital, Herlev Ringvej 75, 2730 Herlev, Denmark. E-mail: line.kessel@dadlnet.dk

Abbreviations: RFLP, Restriction fragment length polymorphism. logical denaturation in parallel with that observed in other tissues [1], resulting in an age-related reduction of the transmission of light to the retina [2] and ultimately impaired visual acuity.

The denaturation process leads to the formation of yellow, fluorescent, insoluble, high molecular weight protein aggregates [3]. In vitro [1] and in vivo studies in animals [4] and humans [5] have shown that the denaturation is caused by non-enzymatic glycation of lens proteins.

The fluorescence of aged lens proteins can be assessed in vivo by non-invasive quantitative fluorometry. Lens autofluorescence is increased in diabetic subjects [6] and there is a relation between the long-term glycaemia and the value of lens fluorescence in hu- 
mans [7]. Previous studies have indicated that lens autofluorescence could have a potential for use in population screening for Type II (non-insulin-dependent) diabetes mellitus [8]. However, a considerable interindividual variation between subjects of the same age and glucose metabolic status remains to be explained.

The present twin study was undertaken to disentangle the genetic and environmental influences on lens fluorescence. Because monozygotic twins are genetically identical and dizygotic twins genetically are no more similar than ordinary siblings, who on average share $50 \%$ of their genes, any greater similarity between monozygotic than dizygotic twins could be attributed to genetic factors. The wider scope of the study was to identify mechanisms underlying the variation in susceptibility to glycation-related diabetic complications in general.

\section{Subjects and methods}

Subjects. We examined 59 monozygotic twin pairs and 55 dizygotic same-sex twin pairs, aged 20 to 46 years. Subjects were recruited from the Danish Twin Registry which is a population-based register comprising twins born in Denmark between 1870 and 1996 [9]. Only subjects in self-assessed good health were invited to participate. Exclusion criteria were cataract or other opacities of the refractive media within $2 \mathrm{~mm}$ of the optical axis of the eye. All participants gave informed consent according to the Helsinki Declaration. The study was approved by the regional ethics committee and followed the tenets of the Helsinki Declaration.

Clinical examination. All subjects had an ophthalmological examination including refractioning, visual acuity determination, slitlamp biomicroscopy, and fundus photography. An oral glucose tolerance test (OGTT) was administered using $75 \mathrm{~g}$ glucose after a $12 \mathrm{~h}$ overnight fast. Blood glucose was assessed at 0,30 , and 120 min on whole (capillary) blood samples. Determination of zygosity was based on genetic markers using 9 microsatellite and RFLP markers. Subjects were asked about lifelong smoking habits. Consumption was assessed in units of pack years, equalling 20 cigarettes per day for one year. Information on diabetes mellitus in first and second degree relatives was obtained.

Ocular fluorometry. Lens fluorescence was measured without artificial pupil dilation along the optical axis of the eye using an ocular fluorometer (Fluorotron, Ocumetrics, San Jose, Calif., USA) fitted with an anterior segment adaptor. Fluorescence was measured in intervals of $0.125 \mathrm{~mm}$ giving detailed information on the lens. Excitation was at 430-490 nm and detection at $530-630 \mathrm{~nm}$. Lens fluorescence was calculated as the mean of six values obtained at the anterior lens peak, three from each eye. A computer program by van Best and Larsen (Leiden NL, Copenhagen DK, version 4.6) was used to correct for loss of light due to absorption as described by another study [10]. Measurements were calibrated to an external fluorescein solution. Fluorescence values are reported in fluorescein equivalents (ng f-eq/ml).

Statistical procedures. In order to evaluate the relative importance of genetic and environmental influences on lens autoflu-

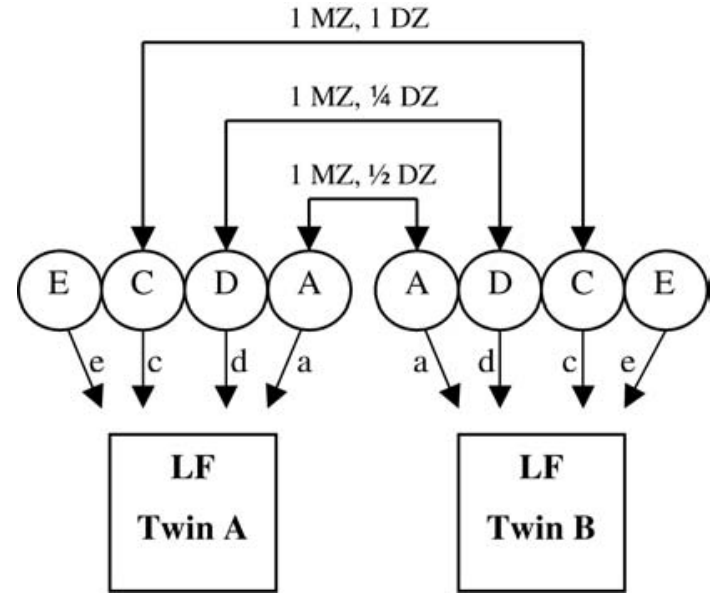

Fig. 1. Path diagram for the structural equation modelling. Interindividual variation in lens fluorescence could be attributable to genetic factors, both additive (A) and non-additive (D), and environmental factors, both those shared by the twins in a pair (C) and those unique to an individual (E). Additive and non-additive genetic effects correlate at 1.0 in $\mathrm{MZ}$ pairs. For DZ pairs a correlation of 0.5 is found for additive genetic effects whereas it is 0.25 for non-additive genetic effects. Lens fluorescence values entered into the models were corrected for the effect of age, glucose metabolism (OGTT at 120 minutes), and smoking habits

orescence structural equation modelling was used to fit different models to the observed data under a number of standard assumptions, i.e. no gene-environment interaction, random mating and equal intra-pairwise environment in monozygotic (MZ) and dizygotic (DZ) twin pairs.

The structural equation modelling approach is based on classical biometric analysis of data. The underlying assumption is that the variance of a phenotype $\left(\mathrm{V}_{\mathrm{P}}\right)$ can be divided into two components, the genotypic variance and the environmental variance. The genotypic variance can be subdivided into two variances, i.e. the variance due to additive genetic effects $\left(\mathrm{V}_{\mathrm{A}}\right)$ and the variance due to non-additive genetic effects $\left(V_{D}\right)$. The additive genetic effects are the effects of genes taken singly and added over multiple loci. The non-additive genetic effects are the effects due to intralocus and interlocus gene interactions. Environmental variance is divided into effects shared by individuals $\left(\mathrm{V}_{\mathrm{C}}\right)$ and effects not shared by individuals $\left(\mathrm{V}_{\mathrm{E}}\right)$, including random error. The shared environmental effects contributes to the phenotypic similarity between individuals and non-shared environmental effects contributes to dissimilarity. The term heritability $\left(\mathrm{h}^{2}\right)$ is used to describe the proportion of the interindividual variation in lens fluorescence attributable to genetic factors, i.e. $\mathrm{h}^{2}=\mathrm{V}_{\mathrm{A}} / \mathrm{V}_{\mathrm{P}}$.

Univariate structural equation modelling was carried out by means of the MX software computer program [11]. The following models were fitted to the data: an ACE model, including additive genetic factors, shared and non-shared environment. The simpler AE and CE models, which assumes that all similarity is a result of additive genetic or shared environmental factors only. Furthermore, two models including non-additive genetic effects were included, the ADE and DE models. The criteria for best fitting model was Akaike's information criterion (AIC), goodness-of-fit chi-square test and root mean square error approximation (RMSEA). The model with the lowest negative AIC reflects the best balance between goodness-of-fit and parsimony. The RMSEA statistics should be 
lower than 0.05 for very good fit or between 0.05 and 0.1 for good fit. The variance components were derived from the models. A path diagram for the structural equation modelling is given in Fig. 1.

Lens autofluorescence data were transformed into logarithmic values to obtain a normal distribution. All fluorescence values reported have been back-transformed to mean values and $95 \%$ confidence interval $(95 \% \mathrm{CI})$. Before lens autofluorescence data was used in the structural equation modelling the data were adjusted for age, smoking and blood-glucose and the residuals of a multiple regression analysis comprising these factors (eq. 1) were entered into the models. Correlations between lens autofluorescence and various factors, such as age, glucose metabolism, and smoking habits, were calculated using Pearsons correlation coefficient. Hypothesis testing was carried out using the Student's $t$ test with a significance level of 0.05 , except if data were not normally distributed, then a non-parametric equivalent was used (Mann-Whitney U test). Comparison of more than two groups was done using an analysis of variance and the $p$-values corrected according to the Bonferroni method. Apart from the genetic analyses, all statistical procedures were carried out using a SyStat computer software (version 7.0 for Windows).

\section{Results}

Lens fluorescence. Lens autofluorescence (LF) was related to an individual's age (A), smoking habits (pack years) (S) and glucose metabolism, measured as 2-h OGTT $(\mathrm{G})$ response (eq. 1, $p<0.001$ ), together these factors were found to account for $60 \%$ (adjusted $\mathrm{R}^{2}$ ) of the variation in lens autofluorescence between individuals:

$L F_{\log 10}=0.016 A+0.005 S+0.025 G-1.730$

Age was the single most important factor for lens autofluorescence, accounting for $53 \%\left(\mathrm{R}^{2}\right)$ of the variation (Table 1). The 2-h OGTT response was found to be closer correlated to lens autofluorescence values than fasting blood glucose. There was no effect of sex on lens fluorescence values, 283.1 (128.2, 625.2) for males versus $290.4(129.1,653.1)$ for females, mean (95\% CI), $p=0.64$. Lens autofluorescence showed no correlation to body mass index $(p=0.5)$ or blood lipids (HDL, VLDL, and triglycerides, $p$ values $>0.1$ ) except for cholesterol $(p=0.009)$. An association was found for lens fluorescence and diastolic blood pressure ( $p=0.001)$ but not for systolic blood pressure $(p=0.4)$.

Smoking was linearly associated with increased lens autofluorescence, $p<0.001$, and the association did not change after a correction for the effect of age by a multiple regression analysis, $p<0.001$. Smokers had fluorescence values of $305.5(132.1,706.3)$ versus $274.2(128.2,586.1)$ for non-smokers, mean $(95 \%$ $\mathrm{CI}), p=0.044$, and smoking was found to account for $10 \%\left(\mathrm{R}^{2}\right)$ of the variation in lens fluorescence (Fig. 2). The difference between smokers and non-smokers was not related to differences in age or in the 2-h OGTTresponse for which there was no difference $(p=0.2$ and $p=0.7$, for age and 2-h OGTT respectively).

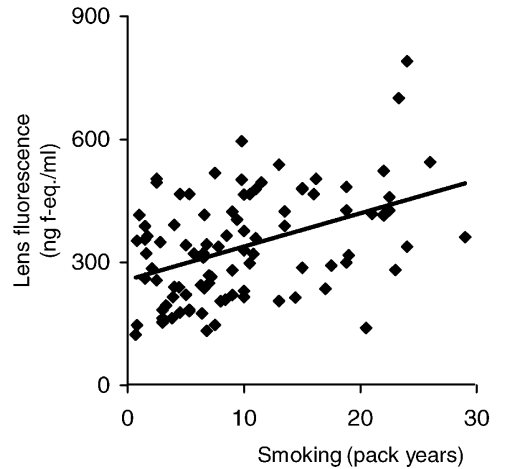

Fig. 2. Lens fluorescence (ng f-eq/ml) in relation to smoking habits (only smokers are shown). The lens fluorescence values presented in the graph are not corrected for the effect of age. A linear regression line has been drawn to show the relation between lens fluorescence and lifelong smoking habits

Table 1. The correlation between lens fluorescence and cofactors

Pearsons correlation coefficient $p$ value ( $95 \%$ confidence interval)

\begin{tabular}{llc}
\hline Age & $0.73(0.66,0.79)$ & $<0.001$ \\
Pack years & $0.31(0.20,0.43)$ & $<0.001$ \\
2-h OGTT & $0.31(0.18,0.42)$ & $<0.001$ \\
Fasting blood glucose & $0.15(-0.02,0.27)$ & 0.03 \\
concentration & &
\end{tabular}

Heritability of lens autofluorescence. No difference was found between the monozygotic and the dizygotic twins in age, glucose metabolism, sex, or smoking habits (Table 2).

The similarity within the MZ twin pairs was found to be greater than within the DZ twin pairs (Fig. 3). For the MZ twins lens autofluorescence values (corrected for the effect of age, OGTT and smoking) of twin A and twin B showed a correlation of 0.84 (95\% CI: $0.78,0.89)$, for the DZ twins the correlation was $0.68(0.51,0.74)$. The structural equation modelling resulted in two significant models: an ACE model, comprising additive genetic effects and shared and non-shared environmental effects, and a CE model, comprising environmental effects only (Table 3). A likelihood-ratio test was used to compare the ACE and $\mathrm{CE}$ model to find out whether the additive genetic effects, A, could be omitted. However, the additive genetic effects were significant and could not be eliminated from the model $\left(\chi^{2}=7.13\right.$, d.f. $\left.=1, p<0.008\right)$. Thus, interindividual variation in lens autofluorescence can be subdivided into the three components of the ACE model: additive genetic effects (the heritability, $\mathrm{h}^{2}$ ) account for $28 \%$ of the variation, shared environment for $58 \%$ and non-shared environment for $14 \%$.

Dividing the twins into a young ( $\leq 36$ years) and old group ( $\geq 37$ years) showed a higher additive genetic ef- 
Table 2. Clinical data for the monozygotic and dizygotic twins

\begin{tabular}{lccc}
\hline & Monozygotic twins & Dizygotic twins & $p$ value (Students $t$ test) \\
\hline Sex (women/men) & $64 / 54$ & $64 / 46$ & \\
Smokers/non-smokers & $47 / 71$ & $52 / 58$ & 0.94 \\
Age in years & $35.1(7.5)$ & $35.1(7.1)$ & 0.34 \\
Lens fluorescence (ng f-eq/ml) & $280.5(134.9,583.4)$ & $295.1(123.6,704.7)$ & 0.99 \\
Fasting-glucose (mmol/l) & $4.9(0.5)$ & $4.8(0.4)$ & 0.86 \\
2-h OGTT (mmol/l) & $6.1(1.3)$ & $6.1(1.3)$ & $0.14^{\mathrm{a}}$ \\
Smoking in pack years & $3.4(5.6)$ & $5.1(7.7)$ & 0.39 \\
Mean arterial blood pressure (mmHg) & $85.3(10.0)$ & $86.3(8.6)$ & 0.25
\end{tabular}

a Mann-Witney U test was used as the numbers of pack years do not follow a normal distribution

Numbers are given in mean (SD), except for lens fluorescence values that are given in mean (95\% confidence interval) after backtransformation from logarithmic values and sex and smoking status that are given in actual numbers

Table 3. The result of the structural equation modelling

\begin{tabular}{|c|c|c|c|c|c|c|c|c|c|}
\hline Model & AIC & $\chi^{2}$ & d.f & $p$ & RMSEA & $a^{2}$ & $\mathrm{D}^{2}$ & $c^{2}$ & $e^{2}$ \\
\hline $\mathrm{ACE}$ & -6.00 & 0 & 3 & 1 & 0 & $\begin{array}{l}0.28 \\
(0.08,0.55)\end{array}$ & 0 & $\begin{array}{l}0.58 \\
(0.32,0.75)\end{array}$ & $\begin{array}{l}0.14 \\
(0.09,0.22)\end{array}$ \\
\hline $\mathrm{AE}$ & 5.21 & 13.21 & 4 & 0.01 & 0.14 & $\begin{array}{l}0.86 \\
(0.80,0.90)\end{array}$ & 0 & 0 & $\begin{array}{l}0.14 \\
(0.10,0.21)\end{array}$ \\
\hline $\mathrm{DE}$ & 20.75 & 28.75 & 4 & 0 & 0.22 & 0 & $\begin{array}{l}0.86 \\
(0.79,0.90)\end{array}$ & 0 & $\begin{array}{l}0.14 \\
(0.10,0.21)\end{array}$ \\
\hline $\mathrm{E}$ & 114.24 & 124.24 & 5 & 0 & 0.62 & 0 & 0 & 0 & 1 \\
\hline
\end{tabular}

Models comprising combinations of genetic effects, both additive (A) and non-additive (D), and environmental effects, both shared (C) and non-shared (E), were tested. The proportion of the total variation in lens fluorescence attributable to additive

fect in the old age group, $42 \%$ (95\% CI: $12,64 \%$ ) versus $18 \%(8,38 \%)$. Furthermore, the effect of the shared environment was reduced, $47 \%(3,73 \%)$ in the older twins versus $80 \%(59,90 \%)$ in the younger twins. The effect of non-shared environment was negligible.

Lens fluorescence was strongly age-dependent and as it is related to advanced glycation end products, formed by non-enzymatic glycation on proteins by glucose or glycotoxins in tobacco smoke, lens fluorescence values were adjusted for the effect of these factors before being entered into the structural equation modelling above. Carrying out the structural equation modelling on lens autofluorescence values not corrected for the effect of age, glucose metabolism and smoking habits resulted in one model that fitted the data: the ACE model. For the uncorrected data additive genetic effects was found to account for $22 \%$ (95\% CI: 12, 37\%), shared environment for $74 \%$ (58, $84 \%)$, and non-shared environment for $4 \%(3,7 \%)$ of the interindividual variation in lens fluorescence. genetic factors (the heritability, $\mathrm{a}^{2}$ ), non-additive genetic factors $\left(\mathrm{D}^{2}\right)$, shared environment $\left(\mathrm{c}^{2}\right)$ and non-shared environment $\left(\mathrm{e}^{2}\right)$ are presented with $95 \%$ confidence intervals

Diabetes. Five twins were found to have diabetes (WHO 1998 criteria [12]) based on either the fasting blood glucose or the 2-h OGTT value. No twin fulfilled both criteria and none had had symptoms of diabetes prior to the study. The five diabetic twins were included in the statistical analysis.

A family history of diabetes in one or more first degree relatives was found in 16 pairs and diabetes in second degree relatives in 12 pairs. The remaining 86 twin pairs had no known family history of diabetes. Family history of diabetes (both Type I and II diabetes) did not influence lens autofluorescence with values of $286.4(130.9,626.6)$ (mean $(95 \%$ CI)) for the twins without diabetic relatives versus 263.6 (120.5, 576.8) for twins with first degree diabetic relatives and $331.1(134.3,816.6)$ for twins with second degree relatives ( $p$ values $>0.05$, analysis of variance with Bonferroni correction). The groups were of the same age and 2-h OGTT-response. 

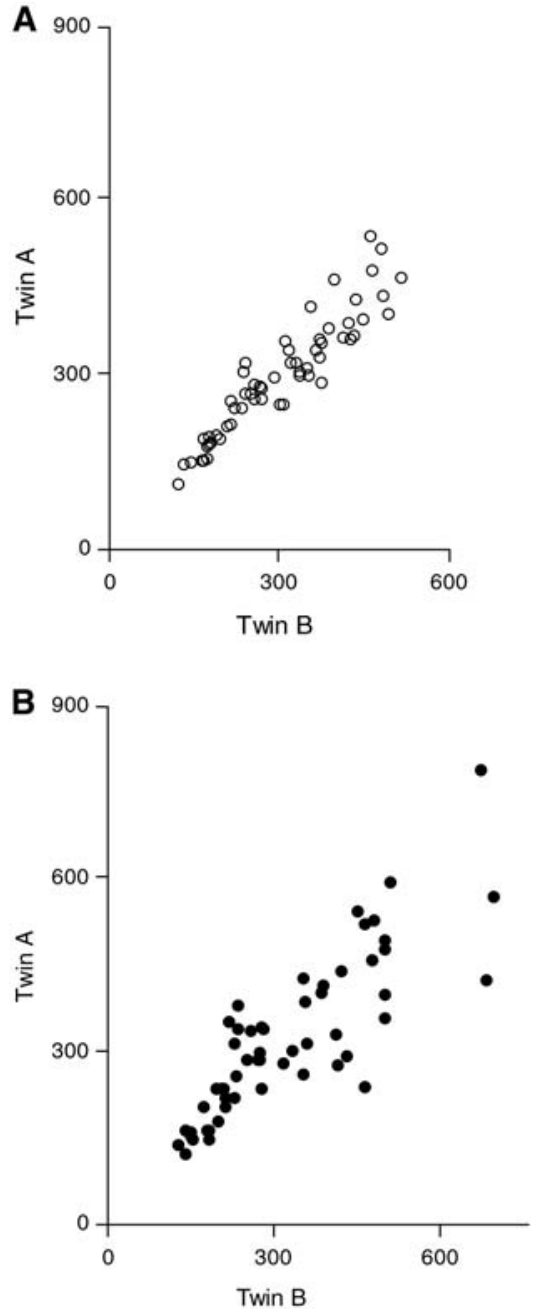

Fig. 3A, B. Lens fluorescence values shown in $\mathrm{ng} f-\mathrm{eq} / \mathrm{ml}$ (not corrected for the effect of age, 2-hour OGTT or smoking habits) for the monozygotic (A) and the dizygotic (B) twins. The value of twin A is plotted against the value of twin $B$

\section{Discussion}

We have examined a type of slow tissue degeneration that is strongly related to diabetes and its long term complications [13] and causes fluorophore accumulation in the lens. We have found that it is partly determined by genetic factors unrelated to current glucose metabolic status or family history of diabetes. The process is accelerated by tobacco smoking. Our finding could help account for the known variation in susceptibility to hyperglycaemia induced tissue and organ damage in diabetic patients, a variation that can not be fully explained by the statistical association to longterm hyperglycaemia and hypertension. This study indicates that a genetic variation in susceptibility to nonenzymatic glycation could explain at least some of the residual variation in the frequency and severity of diabetes.

We found the variation in lens autofluorescence to be a result of the combined effect of additive genetic factors and both shared and non-shared environmental factors. We did not find support for an effect of nonadditive genetic factors which was to be expected based on the high correlation coefficients of both the monozygotic and dizygotic twins. The predominant part of the variation in lens autofluorescence was attributable to shared environmental effects.

The shared environmental effects were surprisingly large in this study and must be attributed to the young age of the twins and the influence of common childhood and possible intra-uterine exposures. We showed a strong correlation between lens autofluorescence and smoking and though the effect of active smoking was accounted for in the analysis, some of the shared environmental effects could be attributable to growing up in a smoking or non-smoking family. Intake of antioxidants have been linked to a decreased risk for developing cataract $[14,15]$; thus, childhood dietary habits could also contribute to the effect of shared environment. Intrauterine factors, such as growth retardation, are known to be important for development of insulin resistance and glucose intolerance in adults [16] and could thus exert an effect on lens autofluorescence in this group of adult twins through the ability to form advanced glycation end products. The hypothesis that common foetal and childhood exposures are important for the amount of fluorophores in the lens of young adults is supported by the observation that the effect of shared environment was reduced in older twins whereas the additive genetic effects became more prominent.

Other aspects of lens ageing have been studied in a genetic perspective $[17,18]$. These studies showed that nuclear cataract, as assessed by subjective visual grading, was related to genetic factors by $35 \%$ and $48 \%$ in the two studies respectively. Although heritability is population specific the overall conclusion is that lens ageing, both the subclinical type examined in this study and cataract-related, is to some degree influenced by genetic factors.

Although modest in size, the genetic effects in our study were statistically significant and of potential clinical relevance. Fluorophore formation in the lens is believed to originate from non-enzymatic glycation of lens proteins. Hypothetically, the genetic influence could be caused by variations in the activity of enzymes modulating the glycated proteins as it has been observed in non-mammalian species $[19,20]$. A study showed that longevity in mammalian species was closely related to the rate of formation of advanced glycation end products and that species able to withstand the tissue damage caused by non-enzymatic glycation had longer life-spans [21]. Taken together, these studies offer support for the idea that the ageing process is related to the formation of advanced glycation end products and that the rate of formation is influenced by genetic factors.

The concerted effect of glucose and smoking provides additional clues to the mechanisms involved in 
lens yellowing and formation of advanced glycation end products. Substances in tobacco smoke can induce advanced glycation end products identical to those formed by glucose [22] and the amount of advanced glycation end products is increased in the lenses and blood of smokers irrespective of diabetes [22, 23]. Smoking has previously been implicated in the pathogenesis of macrovascular complications in Type II diabetic patients [24]. Our result indicate that the effect could be mediated through tissue damage due to nonenzymatic protein denaturation.

Acknowledgements. The authors would like to thank L.K. Brigsted, B. Carstensen and E. Muff Eilersen at the Institute of Preventive Medicine for their assistance. The study was supported by grants from the Novo Nordic Foundation for Research in Biotechnology and Pharmaceutical Sciences, the Danish Diabetes Association, the Danish Heart Foundation, the Danish Medical Research Council and the Danish National Science Foundation (Grundforskningsfonden).

\section{References}

1. Monnier VM, Cerami A (1981) Nonenzymatic browning in vivo: possible process for aging of long-lived proteins. Science 21: 491-493

2. Van Best JA, Vrij L, Oosterhuis JA (1985) Lens transmission of blue-green light in diabetic patients as measured by autofluorophotometry. Invest Ophthalmol Vis Sci 26: 532536

3. Lerman S, Borkman R (1976) Spectroscopic evaluation and classification of the normal, aging, and cataractous lens. Ophthalmic Res 8: 335-353

4. Abiko T, Abiko A, Ishiko S et al. (1999) Relationship between autofluorescence and advanced glycation end products in diabetic lenses. Exp Eye Res 68: 361-366

5. Lyons TJ, Silvestri G, Dunn JA, Dyer DG, Baynes JW (1991) Role of glycation in modification of lens crystallins in diabetic and nondiabetic senile cataracts. Diabetes 40: 1010-1015

6. Bleeker JC, Best JA van, Vrij L, Velde EA van der, Oosterhuis JA (1986) Autofluorescence of the lens in diabetic and healthy subjects by fluorophotometry. Invest Ophthalmol Vis Sci 27: 791-794

7. Larsen M, Kjer B, Dalgaard P, Lund-Andersen H (1989) Lens fluorescence in relation to metabolic control of insulin-dependent diabetes mellitus. Arch Ophthalmol 107: 59-62

8. Theil PK, Hansen T, Larsen M, Pedersen O, Lund-Andersen $\mathrm{H}$ (1996) Lens autofluorescence is increased in newly diagnosed patients with NIDDM. Diabetologia 39: 1524-1527
9. Kyvik KO, Christensen K, Skytthe A, Harvald B, Holm NV (1996) The Danish twin register. Dan Med Bull 43: 467-470

10. Zeimer RC, Noth JM (1984) A new method of measuring in vivo the lens transmittance, and study of lens scatter, fluorescence and transmittance. Ophthalmic Res 16: 246-255

11. Neale MC, Boker SM, Xie G, Maes HH (1999) MX: statistical modelling, 5th edn. Department of Psychiatry, Richmond

12. Alberti KGMM, Zimmet PZ (1998) Definition, diagnosis and classification of diabetes mellitus and its complications. Part 1: diagnosis and classification of diabetes mellitus, provisional report of a WHO consultation. Diabet Med 15: 539-553

13. Larsen M, Kjer B, Bendtson I, Dalgaard P, Lund-Andersen H (1992) Lens fluorescence in relation to nephropathy in insulin-dependent diabetes mellitus. Graefes Arch Clin Exp Ophthalmol 230: 6-10

14. Leske MC, Chylack LT, He Q et al. (1998) Antioxidant vitamins and nuclear opacities. The longitudinal study of cataract. Ophthalmology 105: 831-836

15. Perlman JA, Lyle BJ, Klein R et al. (2000) Vitamin supplement use and incident cataracts in a population-based study. Arch Ophthalmol 118: 1556-1563

16. Barker DJP, Clark PM (1997) Fetal undernutrition and disease in later life. Rev Reprod 2: 105-112

17. Heiba IM, Elston RC, Klein BEK, Klein R (1993) Genetic etiology of nuclear cataract. Evidence for a major gene. Am J Med Genet 47: 1208-1214

18. Hammond CJ, Sneider H, Spector TD, Gilbert CE (2000) Genetic and environmental factors in age-related nuclear cataracts in monozygotic and dizygotic twins. N Engl J Med 342: 1786-1790

19. Saxena AK, Saxena P, Monnier VM (1996) Purification and characterization of a membrane-bound deglycating enzyme (1-deoxyfructosyl alkyl amino acid oxidase EC 1.5.3) from a Pseudomonas sp soil strain. J Biol Chem 271: 32803-32809

20. Takahashi M, Pischetsrieder M, Monnier VM (1997) Isolation, purification, and characterization of amadoriase isoenzymes (fructosyl amine-oxygen oxidoreductase EC 1.5.3) from Aspergillus sp. J Biol Chem 272: 3437-3443

21. Sell DR, Lane MA, Johnson WA et al. (1996) Longevity and the genetic determination of collagen glycoxidation kinetics in mammalian species. Proc Natl Acad Sci USA 93: 485-490

22. Cerami C, Founds H, Nicholl I et al. (1997) Tobacco smoke is source of toxic reactive glycation products. Proc Natl Acad Sci USA 94: 13915-13920

23. Nichol ID, Stitt AW, Moore JE et al. (1998) Increased levels of advanced glycation endproducts in the lenses and blod vessels of cigarette smokers. Mol Med 4: 594-601

24. Turner RC, Millns H, Neil HAW et al. (1998) Risk factors for coronary artery disease in non-insulin dependent diabetes mellitus: United Kingdom prospective diabetes study (UKPDS: 23). BMJ 316: 823-828 